\title{
Predictors of functional recovery for hip fractured elders during 12 months following hospital discharge: a prospective study on a Taiwanese sample
}

\author{
Yea-Ing Lotus Shyu · Min-Chi Chen \\ Jersey Liang $\cdot$ Chi-Chuan Wu $\cdot$ Juin-Yih Su
}

Received: 28 May 2003 / Accepted: 17 November 2003/Published online: 28 February 2004

(C) International Osteoporosis Foundation and National Osteoporosis Foundation 2004

\begin{abstract}
To examine the longitudinal changes in overall and individual physical activities of daily living (PADLs) and instrumental activities of daily living (IADLs), at 1 , 3,6 , and 12 months after hospital discharge in elderly subjects, 110 hip fractured elders (mean $\pm \mathrm{SD}$ age, $79.4 \pm 7.5 ; 60.9 \%$ females) were enrolled in a prospective study. At 12 months following hospital discharge, $56.1 \%$ subjects had recovered their overall PADLs, $37.9 \%$ had recovered their overall IADLs, and $74.2 \%$
\end{abstract}

\footnotetext{
Y.-I.L. Shyu $\bowtie$

Center for Gerontological Research,

Chang Gung University,

259 Wen-Hua 1st Road, Kwei-Shan,

Tao-Yuan 333, Taiwan, ROC

E-mail: yeaing@mail.cgu.edu.tw

Tel.: + 886-3-2118800 ext. 5275

Fax: + 886-3-2118400

Y.-I.L. Shyu

School of Nursing, Chang Gung University,

259 Wen-Hua 1st Road, Kwei-Shan,

Tao-Yuan 333, Taiwan, ROC

M.-C. Chen

Department of Public Health,

Chang Gung University,

259 Wen-Hua 1st Road, Kwei-Shan,

Tao-Yuan 333, Taiwan, ROC

J. Liang

Health Management and Policy,

School of Public Health,

University of Michigan,

M3234 School of Public Health II,

Ann Arbor, MI 48109-2029, USA

C.-C. Wu

Department of Orthopedics,

Chang Gung Memorial Hospital,

5 Fu-Hsin Street, Kwei-Shan,

Tao-Yuan 333, Taiwan, ROC

J.-Y. Su

Department of Trauma \& Emergency Surgery,

Chang Gung Memorial Hospital, 5 Fu-Hsin Street,

Kwei-Shan, Tao-Yuan 333,

Taiwan, ROC
}

could walk independently or with the aide of a cane. When analyzed by generalized estimating equations (GEE), for individual PADL, bathing and climbing stairs had consistently improved at every time point throughout the 1-year follow-up period, while transferring, toileting, and walking ability only improved significantly in the first 3 months after discharge. For individual IADL, the proportion of recovery for mobility appeared to increase significantly during the first 6 months post-discharge, and the remaining IADLs appeared to be stable. These results indicated that the recovery rate is varied for performance of different activities according to the complexity and the involvement of the lower extremities. We also found that less concomitant diseases, and a shorter hospital stay could predict a better recovery trend of overall and of most individual PADLs. These findings may be applicable to other countries with Chinese populations, and could provide a reference for health care providers to develop specific interventions for Chinese hip fractured elders.

Keywords Elderly persons - Health related quality of life $\cdot$ Hip fracture $\cdot$ Longitudinal change

\section{Introduction}

Because of its association with excess mortality of 5$20 \%$, and morbidity that results in huge economic costs, hip fracture is identified as the most dramatic consequence of osteoporosis in the elderly who are 65 years old or older [1]. Previous studies found not only a sustained decline in physical function early after a hip fracture, but also a steady "unrecovery" of a large percentage $(26-76 \%)$ of elderly persons who survive the first or even the second year following a hip fracture [2, $3,4,5,6,7,8,9,10,11,12]$. It is estimated that the incidence rate (per 100,000) of hip fractures for the elderly population in Taiwan in 1993 was 211, and that this number is 10 times the incidence rate for the general population [13]. Despite the use of advanced treatment, 
the mortality rate remains significant, and a majority of the patients will never recover completely in terms of physical activities of daily living (PADLs). In addition, the economic cost of hip fracture in the elderly in Taiwan is huge [13]. As in many other countries in the world, the aging population in Taiwan has increased rapidly, and will continue to grow. The population of Taiwan aged 65 and over is projected to increase from 1.56 million $(7.37 \%)$ in 1994 to 3.4 million $(13.83 \%)$ in 2020 , and 5.6 million $(21.65 \%)$ in the year 2036 [14]. As the aging population increases, the problems caused by hip fracture are aggravated, and it has become a major concern for health care providers in Taiwan.

There is extensive literature addressing the consequences of hip fracture in terms of the impact and recovery in physical functioning $[2,4,6,8,10,11,15$, 16]. However, there are several gaps in the literature. First of all, few previous studies on functional recovery after hip fracture $[11,15,17,18]$ used longitudinal data with more than two time points, and few used methods of longitudinal data analysis to explore the trends and identify risk factors associated with change in functional recovery over time. The trajectory of functional recovery after hip fracture can not only provide information on the consequences of hip fracture at certain points in time, but also on the changes in outcomes from an earlier to a more long-term time period. This information may help health care providers predict the prognosis of the patients more precisely, provide a reference for timing of outcome assessment, and assist them in developing a timely management approach to shape, control or change the course of the functional recovery after hip fracture.

Secondly, prior observations are almost exclusively based on data from Western developed nations, but little is known about the functional recovery of hip fractured elders in Asian countries. It would be critical to evaluate the external validity of these observations with data derived from other societies and cultures. Taiwan differs substantially from the Western countries in health care system, clinical practice, case-mix, culture, and social organization. For example, anthropometrical measurements including body mass index, height, weight, hip axis and femoral neck length are different among different ethnic groups $[19,20,21,22]$. The housing environment in Taiwan is different from that in Western countries. Moreover, the existence of a national health insurance and the choice for people to visit clinics in a medical center without referrals in Taiwan is different from health care systems in Western countries. These differences might make the functional recovery of elders with hip fracture different from their counter parts in Western countries. Thus, the findings from Western countries need to be validated in Taiwan. The similarities and differences between findings from Taiwan and those from Western countries are useful for further scientific understanding and clinical implementation.

This study has two major objectives: a) to describe the return to previous levels of PADLs and IADLs for hip fractured elders during 1 year following their hospital discharge; and b) to explore the effects of hospital stay, concomitant illness and pre-fracture dependency in PADLs on patterns of recovery for overall and individual PADL/IADL. These variables were selected because they were identified as important clinical predictors for prognosis after hip fracture $[11,15]$.

\section{Materials and methods}

A prospective, correlational study design was used to explore the functional recovery following the discharge of hip fractured elders. Data were collected from a sample of elderly persons with hip fracture from a medical center before hospital discharge, and then further at 1, 3, 6 and 12 months after hospital discharge via face-toface interviews at clinics, or in a home setting or via telephone interviews. For those elderly persons who did not return to the clinics and refused to receive home visits, telephone interviews were conducted. For elderly persons who were unable to come to the phone or were cognitively impaired, information from proxy respondents was obtained. Proxies are considered reliable sources of information pertaining to facts and observable behaviors, as reported by Maganizer and colleagues [23].

Study setting and sample

This study was conducted in the trauma wards of a 3800-bed medical center in northern Taiwan and in home settings. The criteria includes was 1) aged 65 years or older; 2) hospitalized due to hip fracture and having received surgery for internal fixation or arthroplasty; and 3) living geographically in northern Taiwan. A total of 110 subjects were enrolled in the study and interviewed before hospital discharge. Eight $(7.3 \%)$ subjects died within 1 month, $5(4.5 \%)$ between the 1st and 3rd month, $2(1.8 \%)$ between the $3 \mathrm{rd}$ and 6 th month, and $3(2.7 \%)$ between the 6th and 12 th month following discharge. One $(0.9 \%)$ subject dropped out before the 1 st month, $4(3.6 \%)$ between the 1 st and 3rd month, $6(5.5 \%)$ between the 3rd and 6th month, and $14(12.7 \%)$ between the 6th and 12th month following discharge due to losing contact caused by changing of address or refusal to participate further.

Procedures

Human subject approval was obtained from the institution before collecting the data. Research assistants identified potential subjects after they received surgery and before they were discharged from the hospital, and invited them to participate in this study. Elderly patients who agreed to participate received face-to-face interviews.

Training programs for interviewers and regular research team meetings were used to monitor the quality of the data obtained. Two registered nurses were hired as interviewers for the study. They attended two training sessions. The first session focused on introduction of the instrument and an interview demonstration. The second training session included an actual interview practice. Problems raised in the data collection were discussed and resolved on a regular basis.

Instruments

\section{Physical functioning}

We used the Chinese Barthel Index (CBI) to assess the physical functioning of hip fracture elders, including dependencies in eating, transferring, grooming, toileting, bathing, walking, climbing stairs, dressing, bowel and bladder control. The CBI was translated by 
Chen et al. [24] and is reported as having established reliability and validity, and as being appropriate for assessing frail elders in Taiwan. The score of the scale ranged from 0 to 100 , with 0 representing total dependence and 100 representing total independence. In this study, Cronbach's alpha of the CBI was 0.87 . We then recoded the score of each item and the total score of the CBI as 1, if the score recovered to the individual's pre-fracture level, and coded it as 0 if the score did not recover to the pre-fracture level.

\section{Instrumental function}

We used the Chinese version of Lawton and Brody's [25] measure for instrumental activities of daily living (IADLs) to assess the instrumental functioning for hip fractures elders, including ability to use the telephone, do shopping, cooking, housekeeping, laundry, transportation, taking medicine and handling money. Lawton and Brody's IADL dependency scale was also translated by Chen and colleagues [24], and is reported as having established reliability and validity, and as being appropriate for assessing frail elders in Taiwan. In this study, a Cronbach's alpha of 0.88 was found. We also examined whether the instrumental function recovered to the individual's pre-fracture level (1) or not (0) in this report.

\section{Predictors of physical function recovery}

Predictors examined in this study included concomitant illness, prefracture walking ability, education (being illiterate or not), whether living with spouse, age, type of surgery, and length of hospital stay. For concomitant illness, the elderly person was considered to have a serious concomitant illness (coded as 1) if any one of the following conditions were noted on their chart: cancer, stroke, heart, renal or liver disease, diabetes mellitus, osteoporosis, or dementia. For pre-fracture walking ability, we used the item for assessing independence in the CBI to assess the pre-fracture walking ability. These variables were included because they were significant in the univariate analysis, and were consistent predictors for functional recovery in many previous studies $[11,15]$.

\section{Data analysis}

The logistic regression was first used to explore the predictors of recovery at each time point (3rd, 6th and 12th month after hospital discharge). The possibility of recovery was modeled as a function of illiterate, age, hospital stay, concomitant disease, pre-fracture walking ability, living with spouse and type of surgery. The significant predictors were further included in the longitudinal analysis.

The generalized estimating equations (GEE) [26, 27] approach was used to determine the risk factors related to the probabilities of hip fractured elders recovering their living ability baseline (1st month) at different time points (3rd, 6th and 12th month after hospital discharge). The GEE approach is able to account for possible correlations in repeated measures over time and to explore the differences among different time points. In contrast with repeated measures with the ANOVA approach, one advantage of using the GEE model is that partial information can also be utilized. That is to say, for those who drop out during the study period, their information before dropping out has made a contribution to the estimating of the parameters relative to the available data. Therefore, it is especially useful for longitudinal studies where sample attrition is an inevitable issue. Its application in our study was to model dependent dichotomous variables (recovered or not in overall PADLs and individual PADL) as a function of time, hospital stay, whether being illiterate or not, type of surgery and concomitant diseases. These predictors were selected because they were significant for overall PADL in the cross-sectional logistic analysis. Because none of the predictors for the overall IADL was significant in the cross-sectional analysis, we modeled recovery of overall IADL and IADL simply as a function of time in the GEE approach. In our study, the physical functioning at 1 month after hospital discharge was used for comparison instead of the prefracture physical functioning as a baseline, since the pattern of recovery after hip fracture was specifically of interest and data after hip fracture were collected prospectively, whereas pre-fracture functioning was collected retrospectively after hospital admission.

For the elders who died $(n=18,16.4 \%)$ or dropped out $(n=25$, $22.7 \%$ ) within a year following discharge, the data before death or dropping out was used for the analysis. The largest number of subjects that dropped out occurred during the 6th month to the 12 th month after discharge. We found that there is little or no difference between the group that pass away or dropped out during the 6th month to the 12th month and the group that stayed on throughout the full 12 months after discharge. In other words, we modeled the dependent binary variables (recovered or not) as function of "time" (baseline, 3rd month, 6th month), "group" (pass away or dropped out between 6 and 12 months after followup versus completed in the 12 months of follow-up) and interaction between time and "group." Note that time is treated as a categorical variable-"baseline," "3rd month" and "6th month." Hence, the interaction between "time" and "group" includes two terms, that is 3rd month $\times$ group and 6th month $\times$ group. It was found that (i) the non-significant interactions indicating the trend of recovery over time were similar in two groups $(P=0.70,0.30$ for eating; $P=0.52,0.71$ for transferring; $P=0.67,0.18$ for grooming; $P=0.65,0.58$ for toileting; $P=0.26,0.96$ for bathing; $P=0.55,0.14$ for walking; $P=0.91,0.59$ for climbing stairs; $P=0.18,0.45$ for dressing; $P=0.76,0.74$ for bowel control; $P=0.23,0.18$ for bladder control; $P=0.73,0.02$ for overall IADL); (ii) after removing interaction, the "group" effect was also ignorable except for bladder control $(P=0.16$ for eating; $P=0.76$ for transferring; $P=0.68$ for grooming; $P=0.63$ for toileting; $P=0.45$ for bathing; $P=0.18$ for walking; $P=0.94$ for climbing stairs; $P=0.47$ for dressing; $P=0.07$ for bowel control; $P=0.05$ for bladder control; $P=0.26$ for overall IADL). We also carried out a parallel analysis using the GEE approach to explore the trends of recovery in PADLs and IADLs only for subjects who remained in the study throughout the whole 12 months. The trends were very similar to the trends for recovery in PADLs and IADLs for the whole sample. As a result, individuals with incomplete information were included for our analysis.

\section{Results}

\section{Proportion of functional independence}

In the original sample $(n=110)$, there were $67(60.9 \%)$ females and 43(39.1\%) males. The age of the subjects ranged from 65 to 96 with a mean age of $79.4(\mathrm{SD}=7.5)$ years, among them, $54(49.1 \%)$ aged 80 or over. Thirtyseven $(33.6 \%)$ of the subjects were married, and 73 $(66.4 \%)$ were widowed. Seventy-eight $(70.9 \%)$ had no formal education, $18(16.4 \%)$ completed primary school (1-6 years), $12(10.9 \%)$ completed high school (7-13 years), and $2(1.8 \%)$ had a completed college $(>13$ years) education. Before the admission, $102(92.8 \%)$ elderly persons lived with their family members, one $(0.9 \%)$ lived with friends, five $(4.5 \%)$ lived in an institution and two (1.8\%) lived alone. Seventy (63.6\%) subjects had received internal fixation and 40 (36.4\%) received arthroplasty. Average length of hospital stay was $12.40(\mathrm{SD}=5.43)$ days; among them, $21(19.1 \%)$ stayed in the hospital for over 2 weeks.

Before their fracture $(n=110), 90(81.8 \%)$ of these elderly patients were fully independent in PADLs, 34 
$(30.9 \%)$ in IADLs, and $106(96.4 \%)$ could walk independently or with the aid of a cane. In contrast, at 1 month following hospital discharge, for the remaining subjects $(n=100)$, only four $(4 \%)$ were fully independent in PADLs, one $(1 \%)$ in IADLs and $45(45 \%)$ could walk independently or with the aid of a cane. At the 3rd month following hospital discharge, for the remaining subjects $(n=91), 12(13.2 \%)$ were fully functionally independent in PADLs, three $(3.3 \%)$ in IADLs and 60 $(65.9 \%)$ could walk independently with the aid of a cane. At 6 months following hospital discharge, for the remaining subjects $(n=83), 21(25.3 \%)$ were fully functionally independent in PADLs, eight $(7.3 \%)$ in IADLs and $55(66.3 \%)$ could walk independently with the aid of a cane. At 12 months following hospital discharge, for the remaining subjects $(n=66), 34(51.5 \%)$ were fully functionally independent in PADLs, two $(3.0 \%)$ in IADLs and $48(72.7 \%)$ could walk independently or with the aid of a cane.

Recovery proportion of individual PADLs and IADLs

The longitudinal changes in recovery proportion of individual PADL and IADL are presented in Fig. 1. It appeared that hip fracture had more impact on activities involving the lower extremities, such as climbing stairs, transferring, bathing, walking and toileting. The longitudinal changes in individual PADL were evaluated by a GEE model (Table 1). Some individual PADLs, including grooming and bowel and bladder control, were quite stable over the 1-year follow-up period. Bathing and climbing stairs consistently improved at every time point throughout the 1-year follow-up period. Several PADLs, including eating, transferring, toileting, and walking ability, only improved significantly in the first 3 months after discharge. Dressing improved significantly from 1 to 6 months, and remained stable from the 6th until the 12th month. The proportion of functional recovery at each time point for each individual PADL is listed in Table 1 . By the end of 12 months, over $75 \%$, in most instances over $80 \%$, of the subjects fully recovered to their pre-fracture functional level in most of the individual PADLs, except for activities involving lower extremities such as toileting, walking and climbing stairs. A little more than $70 \%$ of the subjects were able to recover to their pre-fracture ability in performing toileting, and walking. The poorest recovery proportion was found regarding the climbing of stairs. Less than $60 \%$ of the subjects fully recovered their ability in climbing stairs.

The longitudinal changes of individual IADL are presented in Fig. 2. It appeared that hip fracture had more impact on activities involving the lower extremities, such as shopping and mobility. The recovery proportion of mobility appeared to increase significantly during the first 6 months post-discharge. The ability to use a telephone, take medication, or manage money was less impacted by hip fracture. However, the ability to manage money appeared to decline at the 12th month post-discharge, and the ability to take medication seemed to decline at the 6th month post-discharge. The proportion of functional recovery at each time point for each individual IADL is listed in Table 2. By the end of 12 months, over $70 \%$ of the subjects were able to fully regain their pre-fracture functional level in activities that did not involve the lower extremities. For activities involving the lower extremities, including shopping and mobility, around $50-60 \%$ of the subjects fully recovered.

\section{Recovery proportion of overall PADLs and IADLs}

Longitudinal changes of proportion of elders returning to their pre-fracture levels of overall PADLs, and IADLs are shown in Fig. 3. Only four (4.0\%) patients had regained their pre-fracture overall PADLs, and 31 $(39 \%)$ had recovered their overall IADL performance level, at one month after discharge. At 3 months following hospital discharge, $14(13.2 \%)$ patients had recovered their overall PADLs, and $36(39.6 \%)$ had recovered their overall IADLs performance level, among the remaining subjects $(n=91)$. At 6 months following hospital discharge, $24(28.9 \%)$ patients had recovered their PADLs and $35(42.2 \%)$ had recovered their overall
Fig. 1 Longitudinal changes in individual PADL

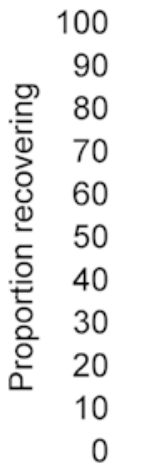
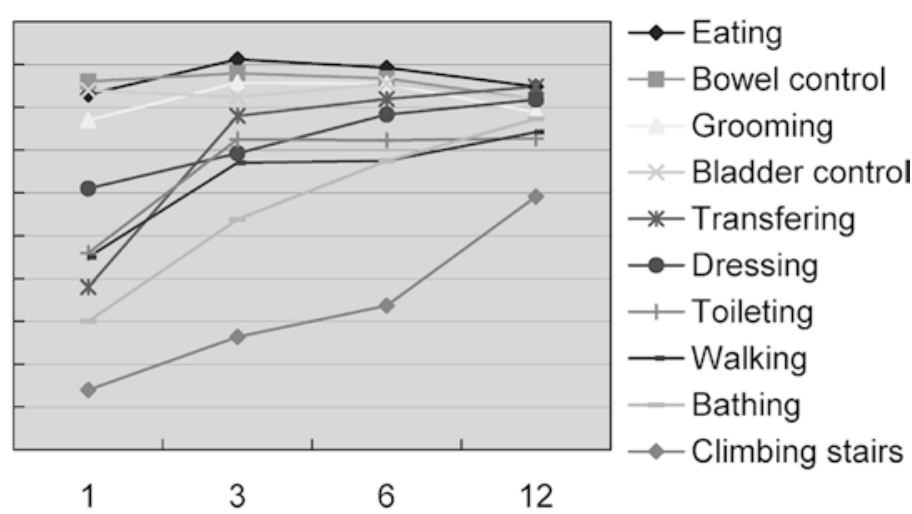

Months following hospital discharge 
Table 1 The number of functional recovery and the total number of observed subjects at each time point for each PADL subscale. The significance in "Time" denotes the difference between the 1st month (baseline) and the 3rd and 6th months and 1 year, respectively. Co-variates entered included hospital stay, whether being illiterate, type of surgery and concomitant disease

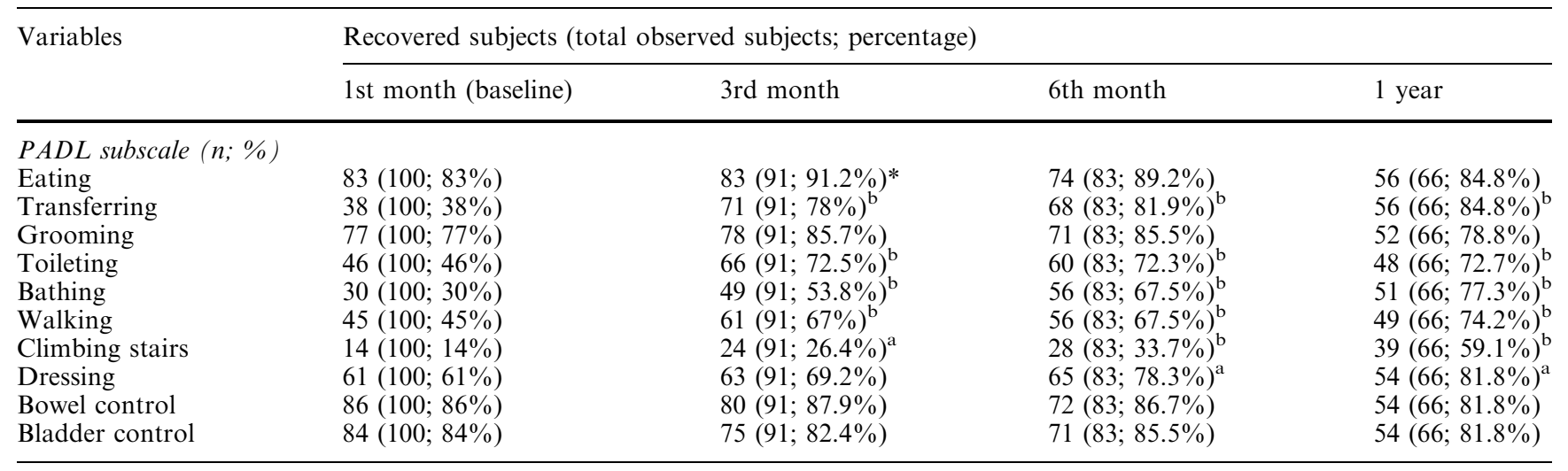

* $P<0.05 ;{ }^{\mathrm{a}} P<0.01 ;{ }^{\mathrm{b}} P<0.001$

Fig. 2 Longitudinal changes in individual IADL
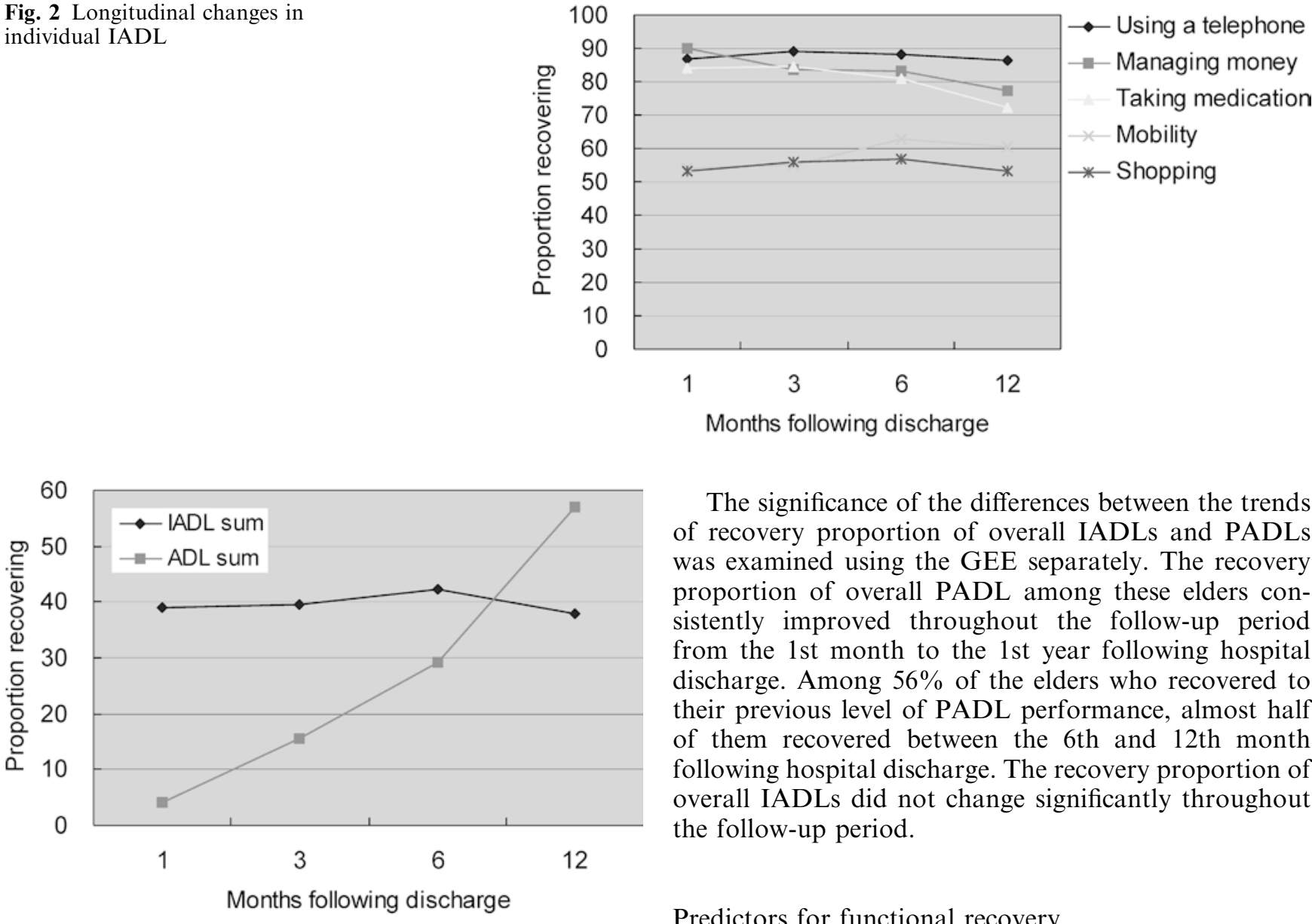

Fig. 3 Longitudinal changes in patient's functional recovery by overall PADLs and IADLs

IADLs performance level among the remaining subjects $(n=83)$. At twelve months following hospital discharge, $37(56.1 \%)$ patients had recovered their overall PADLs and $25(37.9 \%)$ had recovered their overall IADLs performance level, among the remaining subjects $(n=66)$.
The significance of the differences between the trends of recovery proportion of overall IADLs and PADLs was examined using the GEE separately. The recovery proportion of overall PADL among these elders consistently improved throughout the follow-up period from the 1st month to the 1st year following hospital discharge. Among 56\% of the elders who recovered to their previous level of PADL performance, almost half of them recovered between the 6th and 12th month following hospital discharge. The recovery proportion of overall IADLs did not change significantly throughout the follow-up period.

\section{Predictors for functional recovery}

The cross-sectional logistic regressions modeled the PADL at each time point. For the overall PADL, the predictors for recovery at the 1st month after discharge could not be calculated because too few subjects $(n=4)$ had recovered to their pre-fracture level of PADL. Whether living with a spouse, with at least a concomitant disease, with independent pre-fracture walking ability, being illiterate, with hospital stay longer than 14 
Table 2 The number of functional recovery and total observed subjects at each time point for each IADL subscale. The significance in "Time" denotes the difference between the 1st month (baseline) and 3rd, 6th months and 1 year, respectively

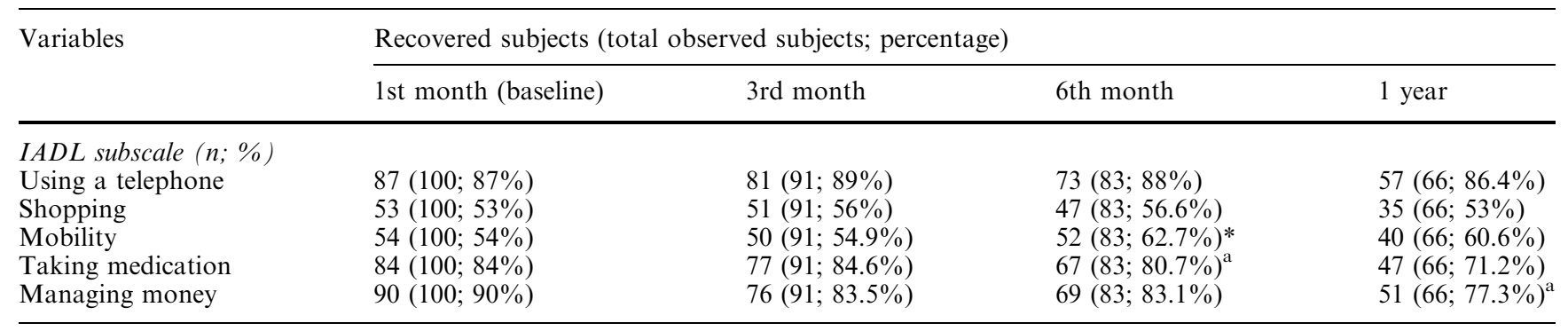

$* P<0.05 ;{ }^{\mathrm{a}} P<0.01$

Table 3 Predictors for recovery of overall PADL at 3rd, 6th and 12th month after discharge. Variables entered were illiterate, age, hospital stay, concomitant disease, pre-fracture walking ability, with spouse and type of surgery. OR followed by $95 \%$ confidence interval in each cell

\begin{tabular}{|c|c|c|c|}
\hline Variables & $\begin{array}{l}\text { 3rd month odds ratio } \\
\text { (confidence interval) }(n=90)\end{array}$ & $\begin{array}{l}\text { 6th month odds ratio } \\
\text { (confidence interval) }(n=82)\end{array}$ & $\begin{array}{l}\text { 12th month odds ratio } \\
\text { (confidence interval) }(n=65)\end{array}$ \\
\hline Illiterate $($ yes $=1 ;$ no $=0)$ & $14.629 *(1.402-152.594)$ & $1.338(0.410-4.370)$ & $1.406(0.434-4.55)$ \\
\hline Age & $0.903(0.808-1.009)$ & $0.951(0.877-1.031)$ & $0.967(0.892-1.048)$ \\
\hline Hospital stay $(\leq 14=1 ;>14=2)$ & $5.404(0.576-50.676)$ & $2.574(0.591-11.210)$ & $5.799 *(1.288-26.108)$ \\
\hline Concomitant disease $($ no $=1 ;$ above $1=0)$ & $3.093(0.746-12.820)$ & $3.787 *(1.263-11.349)$ & $2.645(0.848-8.253)$ \\
\hline $\begin{array}{l}\text { Pre-fracture walking ability } \\
(\text { non-independent }=1 \text {; independent }=0)\end{array}$ & $3.917(0.203-75.496)$ & $1.938(0.130-28.957)$ & $0.909(0.038-21.547)$ \\
\hline With spouse $($ yes $=1 ;$ no $=0)$ & $1.864(0.350-9.939)$ & $0.733(0.235-2.286)$ & $1.031(0.307-3.466)$ \\
\hline $\begin{array}{l}\text { Type of surgery } \\
\text { (internal fixation }=1 \text {; arthroplasty }=0 \text { ) }\end{array}$ & $11.197 *(1.691-74.133)$ & $2.919(0.933-9.134)$ & $2.755(0.891-8.522)$ \\
\hline
\end{tabular}

$* P<0.05$

days, receiving internal fixation versus arthroplasty and age were entered as predictors for recovery of overall PADL (Table 3). At 3 months following discharge, when controlling for other variables, the odds of recovering to pre-fracture level in overall PADLs for subjects who received internal fixation was 11.20 times of that for those who received arthroplasty, and for subjects who were illiterate it was 14.63 times that compared to those who were literate. At 6 months following discharge, the odds of recovery for those who had no concomitant disease was 3.79 times that of those who had at least one concomitant disease. At one year following discharge, the odds of recovery for those who had a hospital stay of less than or equal to 14 days were 5.80 times that of those who had a hospital stay longer than 14 days. In summary, the possibility of recovery is higher for those who are illiterate and received surgery for internal fixation, have no concomitant disease, and have shorter hospital stays at the 3rd, 6th and 12th months after discharge, respectively.

In addition, we used the GEE approach to examine the predictors of recovery trends in overall and individual PADL across different time points. We did not use the GEE approach to examine the predictors of recovery trends in IADLs, because these predictors were not significant in the cross-sectional analysis. In this longitudinal data analysis for recovery trends of overall PADLs and individual PADLs, we entered variables that were significant in the cross-sectional data analysis as predictors including length of hospital stay, concomitant diseases, whether being illiterate or not, and type of surgery. We found that after controlling for other variables, elderly persons who had no serious concomitant diseases appeared to have a better recovery trend of overall PADLs, eating, transferring, grooming, toileting, climbing stairs, and bowel and bladder control than those who had at least one serious concomitant disease. For hospital stay, subjects who had more than 14 days of hospitalization appeared to have a poorer recovery trend of transferring, grooming, toileting, bathing, climbing stairs, dressing, bowel and bladder control, and overall PADLs. In terms of the type of surgery, subjects who received arthroplasty appeared only to have a poor recovery trend in climbing stairs. Type of surgery could not predict the trend of recovery for overall PADLs and other individual PADLs. Subjects who were illiterate did not differ significantly in terms of trends of recovery in individual and overall PADLs.

\section{Discussion}

As one of the most severe consequences of osteoporosis, a large percent (26-76\%) of elderly persons who survive the first or even the second year following hip fracture 
not being able to fully recover to their previous functional level has been reported in Western countries [2, 3, $4,5,6,7,8,9,10,11,12]$. Similarly, in this study with a sample from Taiwan, we found that by the end of the first year, $56.1 \%$ of hip fractured elders fully recovered their pre-fracture level of PADL, 37.9\% had recovered their overall IADLs, and $74.2 \%$ of these elders fully recovered their pre-fracture walking ability. Some studies in Western countries reported the changes over time for the functional recovery after hip fracture [11, $15,18]$, but no such reports have been made yet for Chinese elders with hip fracture. The patterns of longitudinal changes in recovery of overall and individual PADLs and IADLs in a sample from Taiwan were identified in this study.

Several findings of this study were similar as in previous studies. The recovery rate in physical functioning is not constant over time, and the impact and recovery for overall PADLs and IADLs, and individual PADLs were varied $[11,15]$. Higher proportions of patients regained their ability to perform physical than instrumental tasks at 1 year post-discharge [3, 11, 28]. This might be due to the fact that instrumental activities are more complex and require not only basic body function, but also overall physical fitness and are influenced by mental/emotional conditions, and thus are more difficult than PADLs to attain pre-fracture level [29]. It might also be due to the fact that routine care for post-surgery hip fractured patients in Taiwan provides only in-hospital physical rehabilitation for recovery of PADLs, but not for IADLs, but not IADLs. This study also found that a higher impact on hip fracture and a more dramatic increase in the proportion of recovery occurred to activities. That is more complex and dependent on leg and hip strength such as walking, climbing stairs, bathing, toileting, shopping and mobility [11].

In terms of changes over time, we found that, similar with previous studies $[4,10,11]$, the recovery of most of the individual PADLs and IADLs that did recover, except bathing and climbing stairs, occurred within 6 months after hospital discharge with eating, transferring, toileting, and walking ability improving significantly in the first 3 months after discharge, and dressing and mobility improving significantly during the first 6 months. The improvement in bathing, climbing and overall PADLs continued significantly throughout the follow-up 1-year period. It seemed that the more difficult and complex the individual activity that could improve over time, the more time it took to recover to pre-fracture level. For example, in a population-based study, the prevalence of elderly persons with impairment in bathing appeared to be higher than dressing, toileting, transferring and eating [30]. It seems that the first 6 months, especially the first 3 months following discharge is the time period that many activities involving the lower extremities had the most improvement. On the other hand, most of the tasks that only improved during the first 3 months had achieved close to $70 \%$ recovery of the pre-fracture level at the 3rd month after discharge, apparently reaching a ceiling that left little room for further improvement. Simply measuring the return to pre-fracture level for functional improvement may hinder the detection of further functional improvement.

We also observed the decline of the ability to manage money and to take medication between the 6th and 12th months post-discharge. This might be due to the fact that managing money and taking medicine had a recovery of over $80 \%$ at the 1st month after discharge that had little room for improvement. This might also because helpers take over with instrumental activities following the return home, and provide assistance beyond the point of actual need, which may create dependencies and cause functional declines between the 6th and 12th months post-discharge [11, 28].

In terms of predictors for functional recovery, both in cross-sectional analysis and longitudinal analysis, less concomitant diseases, and a shorter hospital stay were found to be important in predicting the overall and most of the individual PADL recovery, and this was also supported by the literature $[4,6,8,10,11,15,18,31]$. Through the cross-sectional analysis, we found that earlier physical function recovery might be influenced by the concomitant disease, and more long-term physical function recovery might be influenced by the length of the hospital stay.

The results indicated that for hip fractured elders in Taiwan, the recovery of physical function involving lower extremities took place in the first 6 months and could last till 1 year after discharge for some complex activities. Based on these findings, several suggestions can be made for clinical practice in taking care of Chinese hip fractured elders. First of all, the recovery rate varies when it comes to the performance of different activities when the complexity and the involvement of lower extremities are taken into consideration. Health care professionals need to pay attention to the variation of the recovery pace for different activities. Secondly, it can be very beneficial to provide rehabilitation programs targeting activities involving lower extremities for up to 6 months, and rehabilitation targeting more complex activities up to 12 months after hospital discharge. Thirdly, more attention and intervention may need to be paid to the high-risk groups for non-recovery, including patients who have concomitant diseases and those who had a longer hospital stay.

In summary, the longitudinal changes of recovery in PADL and IADL of hip fractured elders were explored for a Taiwanese sample. The use of a convenience sample and a certain percentage of subject loss were the only limitations of this study. Several issues raised by the study results can be further explored. For example, does the specific living environment impede the recovery of more complex activities such as bathing and climbing stairs for hip fractured elders in Taiwan? The decline of certain functions after 1 year will need to be further verified. Not only measuring the return to the pre-fracture level, but also more sensitive measures for functional improvement may need to be implemented 
longitudinally in future studies to obtain more detailed information on the trends of functional recovery. Interventional studies based on the findings of this study could also be conducted afterwards. As the population component of elderly Asians is increasing rapidly in many Western countries [32], the results of this study may be applicable to other countries with Chinese populations, and can provide a reference for health care providers in these countries who have to deal with Chinese/Taiwanese immigrants.

Acknowledgment This work was funded by the National Health Research Institute, Republic of China.

\section{References}

1. Randell AG, Nguyen TV, Bhalerao N et al. (2000) Deterioration in quality of life following hip fracture: a prospective study. Osteoporos Int 11:460-466

2. Marottoli RA, Berkman LF, Cooney LM Jr (1992) Decline in physical function following hip fracture. J Am Geriatr Soc 40:861-866

3. Jette AM, Harris BA, Cleary PD et al. (1987) Functional recovery after hip fracture. Arch Phys Med Rehabil 68: 735-740

4. Katz S, Ford AB, Heilpe KG et al. (1964) Studies of illness in the aged: recovery after fracture of the hip. J Gerontol 19:285293

5. Kreulzfeldt J, Haim M, Bach E (1984) Hip fracture among the elderly in a mixed urban and rural population. Age Ageing $13: 111-119$

6. Thomas TG, Steven RS (1974) Social effects of fracture of the neck of the femur. BMJ 3:456-458

7. Jensen JS, Tøndevold E, Sørensen PH (1980) Costs of treatment of hip fractures. Acta Orthop Scand 51:289-296

8. Cobey JC, Cobey JH, Conant L et al. (1976) Indicators of recovery from fractures of the hip. Clin Orthop 117:258-262

9. Mossey JM, Mutran E, Knott K et al. (1989) Determinants of recovery 12 months after hip fracture: the importance of psychosocial factors. Am J Pub Health 79:279-286

10. Jensen JS, Bagger J (1982) Long-term social prognosis after hip fractures. Acta Orthop Scand 53:97-101

11. Magaziner J, Simonsick EM, Kashner TM et al. (1990) Predictors of functional recovery one year follow hospital discharge for hip fracture: a prospective study. J Gerontol 45:M101-107

12. Norton R, Butler M, Robinson E et al. (2000) Declines in physical functioning attributable to hip fracture among older people: a follow-up study of case-control participants. Disabil Rehabil 22:345-351

13. Tsai YJ, Lin HS, Chow LP (1995) A pilot study on health sector priority review in Taiwan: by retrospective study on the elderly hip fracture (monograph). Taiwan Provincial Institute of Family Planning, Taichung, Taiwan
14. Manpower Planning Department, Council for Economic Planning and Development, Executive Yuan, Republic of China (1996) Projections of the population of Taiwan area, Republic of China 1995 to 2036. The Department, Taipei, Taiwan

15. Young Y, Brant L, German P et al. (1997) A longitudinal examination of functional recovery among older people with subcapital hip fractures. J Am Geriatr Soc 45:288-294

16. Nickens HW (1983) Reviews of factors affecting occurrence and outcome of hip fracture, with special reference to psychosocial issues. J Am Geriatr Soc 31:166-170

17. Peterson MGE, Allegrante JP, Cornell CN et al. (2002) Measuring recovery after a hip fracture using the SF-36 and Cummings Scales. Osteoporos Int 13:296-302

18. Barnes B, Dunovan K (1987) Functional outcomes after hip fracture. Phys Ther 67:1675-1679

19. Chin K, Evans MC, Cornish J et al. (1997) Differences in hip axis and femoral neck length in premenopausal women of Polynesian, Asian and European origin. Osteoporos Int 7:344-347

20. Launer LJ, Harris T (1996) Weight, height and body mass index distributions in geographically and ethnically diverse samples of older persons. Ad Hoc Committee on the Statistics of Anthropometry and Aging. Age Ageing 25:300-306

21. Wang MC, Aguirre M, Bhudhikanok GS et al. (1997) Bone mass and hip axis length in healthy Asian, black, Hispanic, white American youths. J Bone Miner Res 12:1922-1935

22. Wang J, Thornton JC, Russell M et al. (1994) Asians have lower bone mass index (BMI) but higher percent body fat than do white: comparisons of anthropometric measurements. Am J Clin Nutr 60:23-28

23. Magaziner J, Simonsick EM, Kashner TM et al. (1988) Patientproxy response comparability on measures of patient health and functional status. J Chin Epidemiol 41:1065-1074

24. Chen YJ, Dai YT, Yang CT et al. (1995) A review and proposal on patient classification in long-term care system. Department of Health, Republic of China, Taipei, Taiwan

25. Lawton MP, Brody E (1969) Assessment of older people, selfmaintaining and instrumental activities of daily living. Gerontologist 9:179-188

26. Liang KY, Zeger SL (1986) Longitudinal data analysis using generalized linear models. Biometrika 73:13-22

27. Liang KY, Zeger SL (1993) Regression analysis for correlated data. Annu Rev Public Health 14:43-68

28. Ceder L, Thorngren KG, Wallden B (1980) Prognostic indicators and early home rehabilitation in elderly patients with hip fracture. Clin Orthop 152:173-184

29. Kane RA, Kane RL (1981) Assessing the elderly: a practical guide to measurement. Lexinton Books, Toronto

30. Wu SC, Hsu HC, Chuang YC, Chang MC (1996) Application of functional assessment in estimating long-term care need among non-institutionalized elderly adults in Taiwan. Chin J Public Health 15:533-544

31. Nickens HW (1983) Review of factors affecting occurrence and outcome of hip fracture, with spherical reference to psychosocial issues. J Am Geriatr Soc 31:166-170

32. Williams DR, Wilson CM (2001) Race, ethnicity, and aging. In: Binstock RG, George LK (eds) (2001) Handbook of aging and the social sciences (5th edn). Academic Press, San Diego, pp $160-178$ 